\title{
TAHSP:-
}

The Internet Joưnal of Allied Health Sciences and Practice

A Peer Reviewed Publication of the College of Health Care Sciences at Nova Southeastern University

Dedicated to allied health professional practice and education

http://ijahsp.nova.edu Vol. 12 No. 3 ISSN 1540-580X

\section{Using Debriefing to Foster Task Management among Anesthesiologist Assistant Students}

\author{
Llalando Austin, EdD \\ Program Director, Department of Anesthesia, Nova Southeastern University, Tampa, Florida \\ United States
}

CITATION: Austin L. Using Debriefing to Foster Task Management among Anesthesiologist Assistant Students. The Internet Journal of Allied Health Sciences and Practice. July 2014. Volume 12 Number 3.

\begin{abstract}
Purpose: This study evaluated the effect of video-aided oral debriefing on task management of anesthesiologist assistant students through the use of high-fidelity anesthesia crisis simulation scenarios. This information describes an evaluation of student learning that is valuable in student educational and workplace competency. Method: A total of 18 graduate students in an anesthesiologist assistant program voluntarily participated in two anesthesia simulation scenarios separated by one of two debriefing methods (oral debriefing versus video-aided oral debriefing). Task management scores of the scenarios were assessed through the Anesthetists' Nontechnical Skills system. Task management scores after the pre-intervention scenario were compared to the scores after the post-intervention scenario. The effectiveness of video-aided oral debriefing was studied using a within-subjects analysis. The comparison of video-aided oral debriefing to oral debriefing was examined with a betweensubjects analysis. Results: The results showed a significant improvement in the intervention group from the initial assessment to the subsequent assessment in task management $(p<0.001)$. However, results did not show a statistically significant difference between the two debriefing methods after a second anesthesia crisis scenario $(p=0.21)$. Conclusion: The research study found that task management during anesthesia crisis scenarios improved after video-aided oral debriefing. No difference was found when comparing the improvement in task management between debriefing methods. Therefore, video-aided oral debriefing was comparable to oral debriefing in improving task management in this study.
\end{abstract}

\section{INTRODUCTION}

The overall education of health care providers has been viewed as an imperfect educational training paradigm. This statement is emphasized by Potts and Davis, who suggested, "There is a growing awareness of weaknesses that exist in the education of health care providers."1 Greiner and Knebel from the Institute of Medicine reported that among existing health care providers, their education and evaluation have largely been inadequate to maintain the necessary proficiency that would ensure safe and high-quality care. ${ }^{2}$ Safe and high-quality care requires competence and effective execution of both technical as well as nontechnical skills.

The problem to be addressed by the research study is the relative absence of task management as a nontechnical skill focus and assessment within the education of anesthesiologist assistant (AA) students as future anesthesia care providers. Educational preparation in established health care curricula should include both technical and nontechnical skills training of future health care providers as well as the utilization of established tools for their assessment. As the educational training of anesthesia care providers prepares for medical expertise inclusive of drugs and equipment as well as other forms of clinical knowledge in the form of technical skills, training to ensure knowledge of nontechnical components has not been explicitly featured in anesthesia training programs. ${ }^{3}$ Specific aspects of nontechnical skills, such as the ability to act as part of a team, fostering communication, cooperation, and leadership are seldom found in health education. ${ }^{4}$ Often, a breakdown in these 
nontechnical skill areas (inclusive of task management) contributes to poor patient outcomes. ${ }^{5}$

Task management is applied to the use of skills for organizing resources and required activities to achieve goals. ${ }^{6}$ In previous reports, it was suggested that observational studies and critical incident reporting in clinical settings as well as with patient simulators have identified task management to be one of the major determinants of successful anesthesia crisis management. 3,7 Howard et al stated that task management, like all other nontechnical skills, is not usually attained by anesthesia trainees via standard routine clinical experience and may need to be specifically taught. 8

Previous studies examining the benefits of video-aided oral debriefing (VAOD) focused on technical skills, communication, stress, and decision making. As an example, Chau et al examined the effect of videotaped vignettes on critical-thinking abilities. ${ }^{9}$ Winters et al examined the effect of videotaping as a teaching and learning strategy and its ability to enhance essential skill ability (i.e., technical skills)..$^{10}$ Additionally, Matthews and Viens evaluated the effect of group video testing on basic nursing skills. ${ }^{11}$ These authors showed that although decision making is one component of nontechnical skills, the other components of nontechnical skill assessments (task management, situational awareness, or team working) through VAOD are lacking.

\section{METHODS}

\section{Study Design}

The study utilized a prospective, two-group, repeated-measure pre- and posttest control group design. The study protocol was reviewed and approved by the Nova Southeastern University (NSU) Institutional Review Board and written consent was obtained from all participants.

Figure 1. Study Design

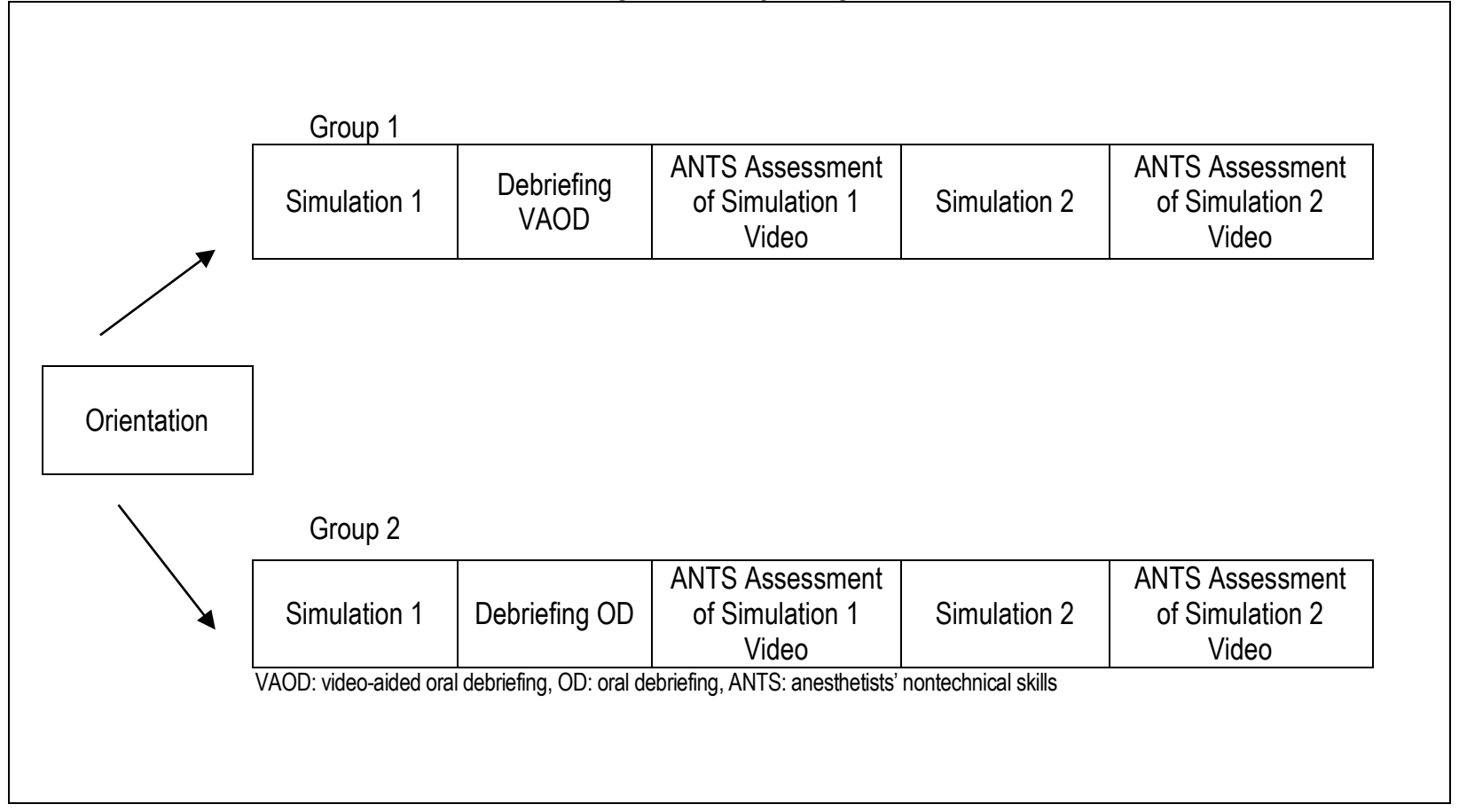

\section{Participants}

Eighteen 2nd-year anesthesiologist assistant students participated in the study. The demographic information was composed of $55.6 \%$ male and $44.4 \%$ female (demographics: 10 male, 8 female; $M_{a g e}=28.11$ years; $S D+5.85$ ). Considering the nontraditional status of many graduate-level students, the ages for all participants ranged from 24 to $4 \overline{4}$ years. 
Table 1. Demographics of Participants

\begin{tabular}{|l|c|c|}
\hline \multicolumn{1}{|c|}{ Demographic } & N & \% \\
\hline Age & & 83.3 \\
\hline $20-29$ & 15 & 11.1 \\
\hline $30-39$ & 2 & 5.6 \\
\hline $40-49$ & 1 & \\
\hline Race/Ethnicity & 3 & 16.7 \\
\hline Black/African American & 13 & 72.2 \\
\hline White/Caucasian & 1 & 5.6 \\
\hline Indian & 1 & 5.6 \\
\hline Asian & & \\
\hline Sex & 10 & 55.6 \\
\hline Male & 8 & 44.4 \\
\hline Female & & \\
\hline
\end{tabular}

\section{Instruments}

The Anesthetists' Nontechnical Skills (ANTS) observation and rating sheet was used as a performance evaluation method for task management of the participants. The ANTS observation and rating sheet was completed by two independent assessors after all simulation scenarios. The two independent assessors had no affiliation with the institution or any of the participants.

The study utilized the ANTS for measurement of the effect of VAOD on task management. The ANTS system was developed by the University of Aberdeen Industrial Psychology Research Centre in collaboration with the Scottish Clinical Simulation Centre. The ANTS system was developed in 1999 when Flin et al obtained funding from the Scottish Council for Postgraduate Medical and Dental Education. ${ }^{12}$ After the completion of preliminary measures to assess usability and acceptability among both consultants and trainees had shown promise, the ANTS system was released in 2004 and made available to anesthesia care providers for noncommercial use at no cost. ${ }^{12}$

The ANTS system includes an observation and rating sheet on which a numerical, scaled value can be assigned to the specified categories of nontechnical skills. Although the observation and rating sheet encompasses four categories, only the task management category was used for data collection. The task management category is composed of four skill elements. The instrument includes a 4-point rating scale ranging from 1 (poor) to 4 (good) for all observed elements with a not observed rating option for specified skills that were not observed.

The reliability and validity for the instrument were examined and noted in previous studies by various authors. Fletcher et al suggested the ANTS system is reliable as a measure of nontechnical skills ability in the simulator environment. ${ }^{6}$ They also found that the ANTS system satisfied aspects of validity. In addition, the results of a slightly more recent study indicated that the ANTS system has construct validity. ${ }^{13}$ In contrast, Flin et al suggested that early reports of the ANTS system content and design in practice were acceptable. ${ }^{12}$ However, they also mentioned that inter-rater reliability was predictably lower when raters had minimal experience and training with the ANTS system. For this reason, it was recommended that a minimum training period of 2 days should be utilized prior to rating participants using the ANTS system. ${ }^{14}$ The independent assessors for this study received their training during the orientation phase over the course of 2 days.

\section{Procedures}

The effectiveness of video-aided oral debriefing was studied using a within-subjects analysis. The comparison of video-aided oral debriefing to oral debriefing was examined with a between-subjects analysis. The research study was carried out in four phases: recruitment, orientation, intervention, and assessment.

\section{Recruitment Phase}

A convenience sampling technique included 26 potential participants, in which 18 agreed to participate. The recruitment phase for the research study was initiated 4 weeks prior to the scheduled date of data collection.

In addition to the participants of the study, three volunteers were asked to participate in the scenarios to role play as members of the intraoperative team: a circulating nurse, a surgical technician, and an AA. Two actively practicing, licensed AAs were recruited to become independent assessors for the evaluation phase. Although active in anesthesia practice, these two independent assessors had no affiliation with the institution or any of the participants involved in the study. The two assessors 
were briefed on the ANTS scoring system and were each provided a copy of the ANTS system handbook to be read in its entirety. Active program-affiliated faculty were asked to volunteer to assist in the operation of the high-fidelity simulation mannequin to guide the progress of the simulated scenarios. All program-affiliated faculty had existing knowledge and experience with high-fidelity mannequin operation and simulated scenario development as well as various debriefing methods.

Once the appropriate consent had been granted, the participants $(N=18)$ were then randomly assigned to two separate groups using a random numbers table, oral debriefing $(O D)(n=9)$ and VAOD $(n=9)$. The control group (OD) was subjected to the existing post-simulation $\mathrm{OD}$ techniques without video-aided assistance. $\mathrm{OD}$ is the manner in which the existing $\mathrm{AA}$ training program implemented post-simulation reflection. The second group was assigned to the experimental group and was exposed to VAOD as the intervention.

\section{Orientation Phase}

All participants and volunteers were instructed on the necessary information to ensure that the research study was able to flow without interruption. Prior to the individual simulation scenarios, a brief 15-minute orientation was held for all participants to discuss anesthesia crisis resource management (ACRM) principles. The basic principles of crisis resource management include environment, resources, reevaluation, attention, teamwork, workload, help, and communication. ${ }^{15}$ Each of these areas was detailed during the brief orientation. Although many ACRM concepts and behaviors were discussed, there was no direct mention of the ANTS scoring system or its affiliated observation and rating sheet.

Those individuals who volunteered to function in the various roles as a part of the perioperative surgical team (circulating nurse, surgical technician, and AA) were also briefed on these roles. These volunteers were not permitted to coerce or provide any information to the subjects during the simulation scenarios and were available only for assistance when it was sought by the study participants. Each participant was briefed on the roles of the surgical team members who were going to be present during the scenario prior to commencing the individual simulation scenarios.

The currently practicing AAs who were recruited to become blinded independent assessors also received an orientation to ensure that the components of the ANTS system, including the handbook and the observation and rating sheet, were thoroughly discussed and understood. The orientation for these volunteers took place over a period of 2 days, as this is the time frame recommended by previous authors who used the ANTS system. ${ }^{14}$ During this period, the underlying psychology and associated anesthesia concerns for task management as a nontechnical skill category mentioned within the ANTS system were discussed. In addition, an explanation of the ANTS system in its entirety was provided.

\section{Intervention Phase}

This phase consisted of two simulation scenarios separated by a debriefing method (OD or VAOD) for each participant. The first anesthesia-crisis scenario focused on the management of pulseless ventricular tachycardia, and the second focused on the management of ventricular fibrillation. These two scenarios were selected as all participants had previous knowledge of the technical management of both. All participants were certified in advanced cardiac life support that covers the discussion and clinical technical-skill management of these scenarios through established protocols. Additionally, all participants had previously undergone rigorous simulation training regarding the management of these scenarios as a component of acquiring their advanced cardiac life-support certification. For the research study, all participants were individually assessed through these scenarios. For each simulation scenario, all participants were required to participate individually, without the assistance of any other study participant.

Participants of both groups (OD and VAOD) were subjected to an initial anesthesia crisis simulation scenario, which focused on the intraoperative management of pulseless ventricular tachycardia while simultaneously being video recorded. Each simulation scenario began with a verbal report and handover of the patient from the role-simulated AA to the individual participant. The provided report included all pertinent information for the scenario including the history for the simulated patient as well as the anesthesia and intraoperative status for the procedure.

The complete crisis-simulation scenario was videotaped for all 18 participants using three cameras, each from different recording views. This three-camera view was overlaid on all videotaped recordings. The three-camera view incorporated two overhead views from different perspectives that focused on the actions of the participants during the simulated scenario. The vital signs of the simulated patient, which were displayed on a 32-inch monitor during the simulation for ease of viewing by the participants, was also graphically displayed and included within the videotaped footage so that viewers of the videotaped footage were able to simultaneously assess the vital signs of the patient as the scenario unfolded. All participants were able to call upon the intraoperative staff present for assistance in managing the scenario but only by asking to perform specific tasks.

(c) The Internet Journal of Allied Health Sciences and Practice, 2014 
Typically, AAs are supervised by an attending anesthesiologist in practice; however, for the purpose of data collection, the anesthesiologist for the scenario was not available, which encouraged the participant to proceed with the simulated management of the patient. Neither the AA nor any other member of the intraoperative staff was able to offer crisis-management advice or contribute to a differential diagnosis. The roles of the intraoperative staff were carried out according to a script. The scenario concluded once the anesthesia crisis-simulation scenario had been adequately managed or at the discretion of the faculty member managing the scenario.

Individual debriefing was carried out immediately after each participant had completed the initial simulation scenario. After completing the first scenario, participants in the experimental (VAOD) group were individually debriefed while simultaneously observing the video recording of their pre-intervention management of the anesthesia crisis-simulation scenario. The participants in the control (OD) group were individually debriefed, albeit only verbally, without the opportunity to observe the video recorded footage of their pre-intervention management of an anesthesia crisis-simulation scenario. Debriefing for both the VAOD and the OD groups was guided by ACRM principles as a component of the simulation experience. The ACRM principles included discussion of environment, resources, reevaluation, workload, teamwork, attention, help, and communication.

The added use of video during VAOD allowed for the ability to pause, fast-forward, or rewind the video-recorded scenarios in order to ensure adequate discussion of scenario-related management and task management discussion between the researcher and each participant during the debriefing phase. There was no limit regarding the number of manipulations of each video recording during the debriefing phase. Therefore, the pause, fast-forward, and rewind features were not used at the same precise points for each participant receiving VAOD.

The control group that received OD did not view any videotaped footage of their scenarios. Oral debriefing was the manner in which the AA students were accustomed to receiving feedback after simulation for the educational training within the anesthesia training program. Each form of post-simulation debriefing focused on nontechnical skill assessments and the associated intraoperative task management. The time allotted for debriefing did not exceed 20 minutes following a 20-minute simulation for each participant. The debriefing was focused on four general areas for reflection: student feelings about the simulation, review of initial assessment steps, review of task management used, and open discussion of individual points of interest. ${ }^{16}$

After completion of the initial anesthesia crisis-simulation scenario followed by debriefing, all participants returned a day later to manage a second anesthesia crisis- simulation scenario. For a second time, the previous volunteers were available to role play as members of the intraoperative surgical team. The second scenario focused on the intraoperative management of ventricular fibrillation as an anesthesia crisis. Again, the surgical team was debriefed on this particular scenario prior to the assessment phase of the scenario. Afterwards, the participants for both OD and VAOD groups were subjected to a post-intervention anesthesia crisis-simulation scenario while simultaneously being video recorded. No debriefing took place for any subject after the culmination of the second scenario, as all necessary study-related data had been collected by this time.

\section{Assessment Phase}

The video recordings of all participants were randomly assigned numbers for identification purposes. All video recordings were forwarded to the two independent assessors for evaluation of the recorded participants through utilization of the ANTS system. The data were then collected and input into Statistical Product and Service Solutions (SPSS) software Version 21 for data analysis.

\section{Data Analysis}

The scores for all participants were calculated and analyzed using SPSS Version 21 to determine the effects of the debriefing methods on task management. A repeated-measure comparison was made to determine the effect of VAOD on task management. Comparison was also made between the VAOD and OD groups with consideration to task management via ACRM simulation scenarios. Descriptive statistics were processed and the distribution of data plotted.

Paired-samples $t$ tests were conducted utilizing task management as the dependent variables. These tests enabled a pre- and post-intervention VAOD assessment of the mean differences for task management. Independent-samples $t$ tests were conducted utilizing task management as the dependent variable. These tests enabled a comparison of the mean differences between the VAOD and OD groups in task management.

\section{RESULTS}

The first research question sought to determine whether debriefing (VAOD or OD) after an initial anesthesia crisis-simulation scenario had a possible effect on task management as a specific nontechnical skill category of AA students after a second

(c) The Internet Journal of Allied Health Sciences and Practice, 2014 
anesthesia crisis- simulation scenario. A paired-samples t test was conducted to evaluate whether participants in the control group improved in task management after the $O D$ intervention. The results indicated that the mean post-intervention score ( $M=$ $3.76, S D \pm 0.23)$ was significantly greater than the mean pre-intervention score $(M=2.35, S D \pm 0.93)$ for task management, $t$ $(17)=6 . \overline{3} 9, p<.001$. A paired-samples $t$ test was conducted to evaluate whether participants in the experimental group improved in task management after the VAOD intervention. The results indicated that the mean post-intervention score ( $\mathrm{M}=$ $3.67, S D \pm 0.41)$ was significantly greater than the mean pre-intervention score $(M=2.31, S D \pm 0.92)$ for task management, $t$ $(17)=9.0 \overline{6}, p<.001$.

The second research question sought to determine whether AA students who received VAOD would score differently on task management assessments than AA students who received only OD after a repeat ACRM scenario. Independent-samples $t$ tests were conducted to evaluate the differences or similarities in post-intervention scores on the dependent variable. For task management as the dependent variable, participants in the $\mathrm{OD}$ group $(\mathrm{M}=3.76, \mathrm{SD} \pm 0.23)$ actually scored higher than participants in the VAOD group $(M=3.59, S D \pm 0.52)$; however, this difference was not statistically significant, $t(34)=1.28, p=$ 0.21 (2-tailed).

\section{DISCUSSION}

This study was not without limitations. As the researcher utilized a convenience sampling method, participants were not randomly sampled, which limited the ability to generalize the process and results of this study. Additionally, the small sample size may have lowered the ability to generalize the findings while increasing sample bias. Although all students maintained an equivalent number of hours and course credits for didactic, laboratory, and clinical training, the amount of time that each participant was exposed to ACRM during the clinical aspect of training may have varied between participants.

The VAOD debriefing technique may have served as a distraction and may have left less time to debrief verbally, as there was only a 20-minute period of time allotted for debriefing. Because the study was held over the course of 2 full days, participants scheduled for earlier times may have affected the study from possible shared discussions among participants of the anesthesia crisis-simulation scenario content. Although the research study sought to determine the effect of the intervention on task management, the participants were aware of their presence in a simulated scenario and, therefore, some aspects of a true anesthesia crisis (e.g., stress) could not be replicated.

The study findings may be beneficial to all stakeholders of training programs in medicine. This study is also beneficial to currently practicing anesthesia care providers including anesthesiologists, certified registered nurse anesthetists, and AAs, as it offers a view of task management as a nontechnical skill and potential techniques for improving task management in a perioperative environment.

This study adds to the existing body of knowledge of clinical simulation training, debriefing methods, and nontechnical skill assessments as they are utilized for educational training among health care professionals. In addition, current practicing anesthesia care providers involved in the use of simulation training for continuing education may benefit as well. Training programs for various health care professions may see a benefit when similar assessments are implemented in their existing educational training curriculum.

\section{CONCLUSIONS}

Despite the demonstrated improvements in task management scores from pretest to posttest, the results of the research study did not indicate a significant difference in task management scores between the two groups. Participants in the VAOD group actually achieved lower scores following debriefing as compared to the OD group, although the difference was not statistically significant. This finding indicated that the quality of task management was not significantly determined by debriefing method but was significantly affected by the presence of debriefing in any form following simulation.

In conclusion, the research study found that task management for ACRM improved after both debriefing methods. However, no difference was found when comparing the improvement in task management performance between debriefing methods. Therefore, VAOD was comparable to OD in improving task management in this study.

\section{REFERENCES}

1. Potts DA, Davis, KF. Making education stick! J Emerg Nurs. 2009;35:375-8. [PMID 19591742]

2. Greiner AC, Knebel E. Health professions education: a bridge to quality. Washington, DC: National Academics Press; 2003.

3. Fletcher GC, McGeorge P, Flin RH, Glavin RJ, Maran NJ. The role of non-technical skills in anaesthesia: a review of current literature. Br J Anaesth. 2002;88:418-29. [PMID 11990277] 
4. Kyrkjebo JM, Brattebo G, Smith-Strom $\mathrm{H}$. Improving patient safety by using interprofessional simulation training in health professional education. J Interprof Care. 2006;20:507-16. doi: 10.1080/13561820600918200. [PMID 17000476]

5. de Leval MR, Carthey J, Wright DJ, Farewell VT, Reason JT. Human factors and cardiac surgery: a multicentre study. J Thorac and Cardiovasc Surg. 2000;119:661-72.

6. Fletcher GC, Flin RH, McGeorge P, Glavin RJ, Maran NJ, Patey R. Anaesthetists' non-technical skills (ANTS): evaluation of a behavioural marker system. Br J Anaesth. 2003;90:580-8. [PMID 12697584]

7. Gaba DM, Fish KJ, Howard SK. Crisis management in anesthesiology. Philadelphia, PA: Churchill Livingstone; 1994

8. Howard SK, Gaba DM, Fish KJ, Yang G, Sarnquist FH. Anesthesia crisis resource management training: teaching anesthesiologists to handle critical incidents. Aviat Space Environ Med. 1992;63:763-70. [PMID 1524531]

9. Chau JP, Chang AM, Lee IF, Ip WY, Lee DT, Wootton Y. Effects of using videotaped vignettes on enhancing students' critical thinking ability in a baccalaureate nursing programme. J Adv Nurs. 2001;36(1):112-9.doi: 10.1046/j.13652648.2001.01948.x. [PMID 11555055]

10. Winters J, Hauck B, Riggs CJ, Clawson J, Collins JW. Use of videotaping to assess competencies and course outcomes. J Nurs Educ. 2003;41:472-6. [PMID 14577736]

11. Matthews R, Viens DC. Evaluating basic nursing skills through group video testing. J Nurs Educ. 1988;27(1):44-6. [PMID 2828577]

12. Flin R, Patey R, Glavin R, Maran N. Anaesthetists' non-technical skills. Br J Anaesth. 2010; 105(1):38-44. [PMID 20522911]

13. Yee B, Naik VN, Joo HS, Savoldelli GL, Chung DY, Houston PL, Karatzoglou BJ, Hamstra SJ. Nontechnical skills in anesthesia crisis management with repeated exposure to simulation-based education. Anesthesiology. 2003;103:241-8. doi: 10.1097/00000542-200508000-00006. [PMID 16052105]

14. Klampfer B, Flin R, Helmreich R, Hausler B, Sexton B, Fletcher G, Field P, Staender S, Lauche K, Dieckmann P, Amacher A. Enhancing performance in high risk environments: recommendations for the use of behavioural markers. Ladenburg: Daimler-Benz Foundation; 2001.

15. Fletcher JL. ERR WATCH: anesthesia crisis resource management from the nurse anesthetist's perspective. J Am Assoc Nurse Anesth. 1998;66:595-602. [PMID 10488267]

16. Chronister C, Brown D. Comparison of simulation debriefing methods. Clin Sim Nurs. 2012;8:e281-8. doi: 10.1016/j.ecns.2010.12.005. 